\title{
Tuning of the Characteristics of Au Nanoparticles Produced by Solid Target Laser Ablation Into Water by Changing the Irradiation Parameters
}

\author{
MARIA KALYVA, ${ }^{1 *}$ GIOVANNI BERTONI,${ }^{2}$ ATHANASIOS MILIONIS,${ }^{1}$ ROBERTO CINGOLANI, ${ }^{2}$ \\ AND ATHANASSIA ATHANASSIOU ${ }^{1,3}$ \\ ${ }^{1}$ Center of Biomolecular Nanotechnologies (CBN), Italian Institute of Technology (IIT), 73010 Arnesano, Lecce, Italy \\ ${ }^{2}$ Nanochemistry Facility, Italian Institute of Technology (IIT), 16152 Genova, Italy \\ ${ }^{3}$ Nanocomposite and Responsive Materials Division, National Nanotechnology Laboratory (NNL) of CNR-Istituto \\ di Nanoscienze, 73100 Lecce, Italy
}

\section{KEY WORDS gold nanoparticles; colloidal solution; laser ablation; transmission electron microscopy; plasmon resonance absorption}

\begin{abstract}
We report the production of Au nanoparticles with different average sizes and size distributions, by laser ablation of a solid Au target into pure deionized water. Tuning laser parameters such as pulse duration, energy, and wavelength is possible to tune the size and the size distributions of the produced nanoparticles into the liquid. We demonstrate the possibility of production of highly monodispersed colloidal solutions, in which the average nanoparticle size ranges from 3 to $10 \mathrm{~nm}$, using laser pulses of ns duration. Laser ablation using fs laser pulses can also produce very small nanoparticles, although a small population of bigger nanoparticles is always present. Low and high-resolution transmission electron microscopy (TEM), in combination with UV-Vis spectroscopy have been employed for the characterization of our samples. Microsc. Res. Tech. 73:937-943, 2010. @ 2010 Wiley-Liss, Inc.
\end{abstract}

\section{INTRODUCTION}

Production and study of nanoparticles (NPs) have attracted considerable interest due to their unique optical, electromagnetic, and catalytic properties, which can be used in many potential technological applications (Alivisatos et al., 1996, Brus et al., 1991, Henglein, 1989). These properties originate from the intrinsic material characteristics, but on the top from their large surface to volume ratio or the fact that interaction with electromagnetic fields can induce coherent oscillations of the conduction electrons (Link and ElSayed, 1999a). The properties of the NPs vary with their size and shape, and this is the reason why much effort has been focused on controlling both (Henglein, 1993, Link and El-Sayed, 1999b). Usually surface stabilizers or templates are used to overcome thermodynamic instability during the NP-formation and determine their final size and shape.

Laser ablation of solid targets into liquids is a quite new alternative physical method for fabrication of NPs (Mafune et al., 2001a; Sibbald et al., 1996). In contrast to the other techniques like chemical synthesis, electrochemical deposition, ablation into vacuum etc., laser ablation in liquid environment is capable to produce metal NPs without using any surface active substances, called also as "green synthesis" (Besner et al., 2008). With this method, can be prepared NPs without chemical surfactants, therefore, having a more direct and reliable response to their surrounding material, increasing the possibility to be used in a wider range of applications.

In particular, for the production of gold $(\mathrm{Au}) \mathrm{NPs}$, diverse groups have applied nanosecond (ns) laser pulses for the ablation of solid Au targets in different solvents, using relatively high energies ( $>7 \mathrm{~mJ} / \mathrm{pulse}$ ), and studying the chemical reactivity of the NPs with the different surrounding media (Compagnini et al., 2002; Mafune et al., 2001b). The size and shape of the NPs have been reported to change by varying the concentration of different surfactants, e.g., decane-dodecanethiol (DDT) solutions reduce the NP-size down to $\sim 1.5 \mathrm{~nm}$ (Compagnini et al., 2006). Usually, most of the studies use pulses with ns duration at wavelengths around $500 \mathrm{~nm}$, i.e., at the $\mathrm{Au}$ plasmon resonance peak, in order to induce further size reduction of the NPs by melting or fragmentation (Bozon-Verduraz et al., 2003). The possibility of producing fine Au particles $<10 \mathrm{~nm}$ by $\mathrm{ns}$ laser ablation in pure deionized water as a single step procedure is under question, while a systematic study of laser ablation by ns pulses at around $500 \mathrm{~nm}$ of an Au solid target in water, varying the laser energy is missing. Studies of laser ablation by ns pulses at 1,064 $\mathrm{nm}$ (Mafune et al., 2001a), are only a few and mainly using surfactants, while in pure deionized water are absent.

In the last few years, generation of NPs by laser ablation into liquids has been attempted also by ultra short pulses. The advantage of this procedure is the reduced melting effects, due to the short duration of the laser pulse compared to the heat diffusion time (Ready and Farson, 2001). Also in this case, the varia-

*Correspondence to: Maria Kalyva, Center of Biomolecular Nanotechnologies (CBN), Italian Institute of Technology (IIT), via Barsanti 1, 73010 Arnesano, Lecce, Italy. E-mail: maria.kalyva@iit.it

Received 12 January 2010; accepted in revised form 20 March 2010

DOI 10.1002/jemt.20868

Published online 6 May 2010 in Wiley Online Library (wileyonlinelibray.com). 
tion of the concentration of different surfactants in the solutions influences the sizes of the produced NPs (Kabashin and Meunier, 2003; Petersen et al., 2009). To change the size and size distribution of NPs many groups use femtosecond (fs) laser ablation of already prepared NPs, e.g., by chemical synthesis (Besner et al., 2006). A systematic work on producing Au NPs into water by fs laser ablation of a solid target was done by changing the focus of the laser beam with respect to the surface of the target (Sylvestre et al., 2005). They showed that for low energies when the focal plane is far/close to the surface target, small NPs with narrow distributions/highly dispersed are produced, respectively. Finally, to get NPs of very small sizes and almost monodispersed, the procedure of $\mathrm{fs}$ laser ablation of an Au target in pure deionized water, was optimized for two different values of laser fluence (one low and the other high), whereas for all the other fluence values higher distributions were obtained (Kabashin and Meunier, 2003).

Herein, is presented a systematic study on the ideal laser parameters for the production of monodispersed $\mathrm{Au} \mathrm{NPs}_{\mathrm{s}}$ in pure deionized water. The laser parameters that were examined are the energy $(E)$, the pulse duration $\left(t_{\text {pulse }}\right)$, and the wavelength $(\lambda)\left(t_{\text {pulse }}=100 \mathrm{fs}\right.$ and $\lambda=800 \mathrm{~nm}, t_{\text {pulse }}=7 \mathrm{~ns}$ and $\lambda=1,064$ or $\left.532 \mathrm{~nm}\right)$. Using laser pulses with fs duration, for laser energies between 0.2 and $0.8 \mathrm{~mJ} /$ pulse, most of the produced NPs have small size $(\sim 2.5 \mathrm{~nm})$ while a minor percentage with larger sizes exists. The bigger particles have increasing sizes for increasing laser energies. The spot diameter was very small and could not be measured, that is why using geometric optics was calculated to be $\sim 80 \mu \mathrm{m}$ and, therefore the range of the fluences used in fs laser ablation is from 3.9 to $16 \mathrm{~J} / \mathrm{cm}^{2}$. When laser pulses of ns duration and $\lambda=532 \mathrm{~nm}$ are used, increasing the pulse energy (from 0.4 to $5.2 \mathrm{~mJ} /$ pulse) monodispersed colloidal solutions are produced with increasing average NP sizes (from 3 to $10 \mathrm{~nm}$ ), whereas at even higher energy pulses $(7.5 \mathrm{~mJ} /$ pulse) a decrease in the average size is observed. The spot diameter was measured $0.4 \mathrm{~mm}$ and, therefore the range of the fluences used in $\mathrm{ns}$ laser ablation is from 0.3 to $5.9 \mathrm{~J} / \mathrm{cm}^{2}$. Finally, when laser pulses of ns duration and $\lambda=1,064 \mathrm{~nm}$ are used, the whole procedure is more complicated since NPs, nanorods, and fragments are produced simultaneously.

\section{MATERIALS AND METHODS}

Gold NPs were prepared by laser ablation of a gold metal rod (dia $9 \mathrm{~mm} \times 6 \mathrm{~mm},>99.99 \%$ ) in ultra pure $18 \mathrm{M} \Omega$ deionized water. The metal rod was placed on the bottom of a glass cuvette filled with $1.5 \mathrm{~mL}$ of pure deionized water, in all experiments. The rod was irradiated with a) a Coherent Legend Ti-Sapphire laser with $\sim 100 \mathrm{fs}$ pulse duration, emitting at $800 \mathrm{~nm}$ and operating at $10 \mathrm{KHz}$ (actual laser-target interaction time: $\left.18.0 \times 10^{-7} \mathrm{~s}\right)$, (b) the fundamental $(1,064 \mathrm{~nm})$ of a Nd:YAG laser (Quanta-Ray GCR-190, Spectra Physics) operating at $10 \mathrm{~Hz}$ and (c) its second harmonic (532 $\mathrm{nm}$ ) with pulse duration $<7 \mathrm{~ns}$ (actual laser-target interaction time: $12.6 \times 10^{-7} \mathrm{~s}$ ), on a rotating base with speed 4.5 degrees/s. The laser beam was focused by a UV-vis (quartz) lens with focal point at $10 \mathrm{~cm}$ for the $\mathrm{ns}$ and by a coated at $800 \mathrm{~nm}$ lens with focal point at $7.5 \mathrm{~cm}$ for the fs laser respectively. In all measure- ments the laser beam was focused $\sim 1 \mathrm{~mm}$ below the surface target, and the irradiation time was kept constant (30 min). Upon irradiation, the solution gradually turned in different experiments from light red to wine red. The absorption spectra of the colloidal solutions were measured immediately after fabrication by a Cary UV-Visible spectrophotometer.

High-resolution transmission electron microscopy (HRTEM) images were acquired from the NPs produced by fs pulses on a JEOL JEM-2200FS operating at 200 $\mathrm{kV}$ and equipped with a spherical aberration corrector in the objective lens, to ensure a point resolution of $1.2 \AA$. Low-magnification transmission electron microscopy (TEM) images of the produced NPs were taken with a Jeol Jem 1011 microscope operating at an accelerating voltage of $100 \mathrm{kV}$. All the samples for TEM analysis were prepared by putting $\sim 40 \mu \mathrm{L}$ of the prepared solution onto carbon-coated 300 mesh, 3-mm copper grids, and then allowing to dry overnight under inert atmosphere. The diameters of more than $500 \mathrm{NPs}$ shown in the TEM images were measured each time for the estimation of the distribution of the NP-diameters (size distribution).

\section{RESULTS}

First, colloidal Au solutions were produced by laser ablation of a solid target, using laser pulses with $\sim 100$ fs pulse duration, emitting at $800 \mathrm{~nm}$, with energies $0.2,0.4,0.6$, and $0.8 \mathrm{~mJ} /$ pulse, respectively. Characteristic of the produced noble metal NPs is the coloring of their colloidal solutions, which is caused by their surface plasmon absorption. In Figure 1 are presented the UV-Vis absorption spectra of all the produced solutions. All absorption spectra appear to have broad bandwidths, with the maximum peak of the one corresponding to the minimum laser energy $(0.2 \mathrm{~mJ} /$ pulse $)$ being between 530 and $535 \mathrm{~nm}$. For higher energies, the absorption maximum shifts to $\sim 540$ and $550 \mathrm{~nm}$. The plasmon absorption maximum and its bandwidth are affected by the size, the shape, and the composition

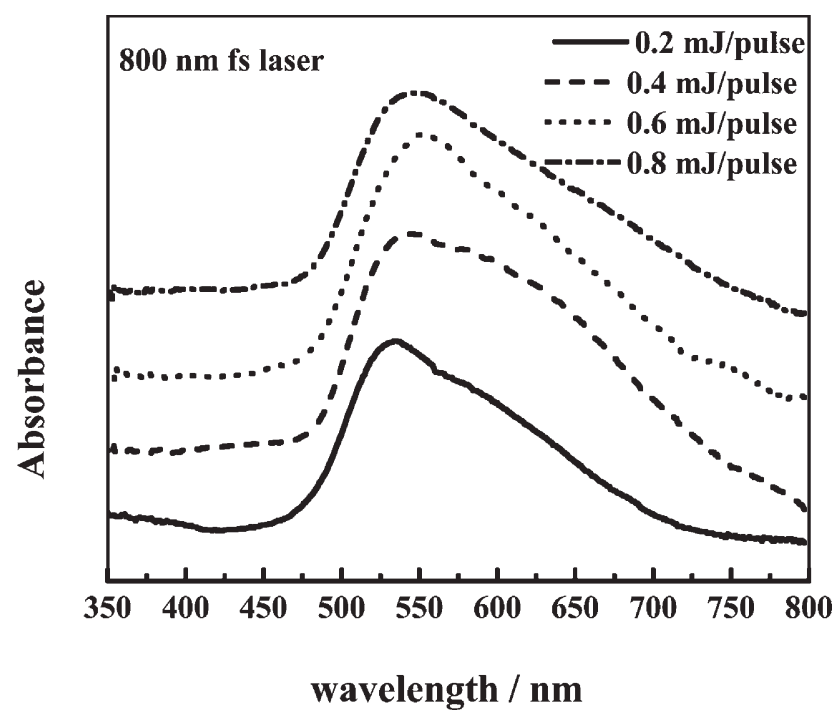

Fig. 1. UV-Vis absorption spectra of the produced Au NPs by laser ablation of solid Au target using pulses with fs duration, wavelength $800 \mathrm{~nm}$, and energies $0.2,0.4,0.6$, and $0.8 \mathrm{~mJ} /$ pulse. 

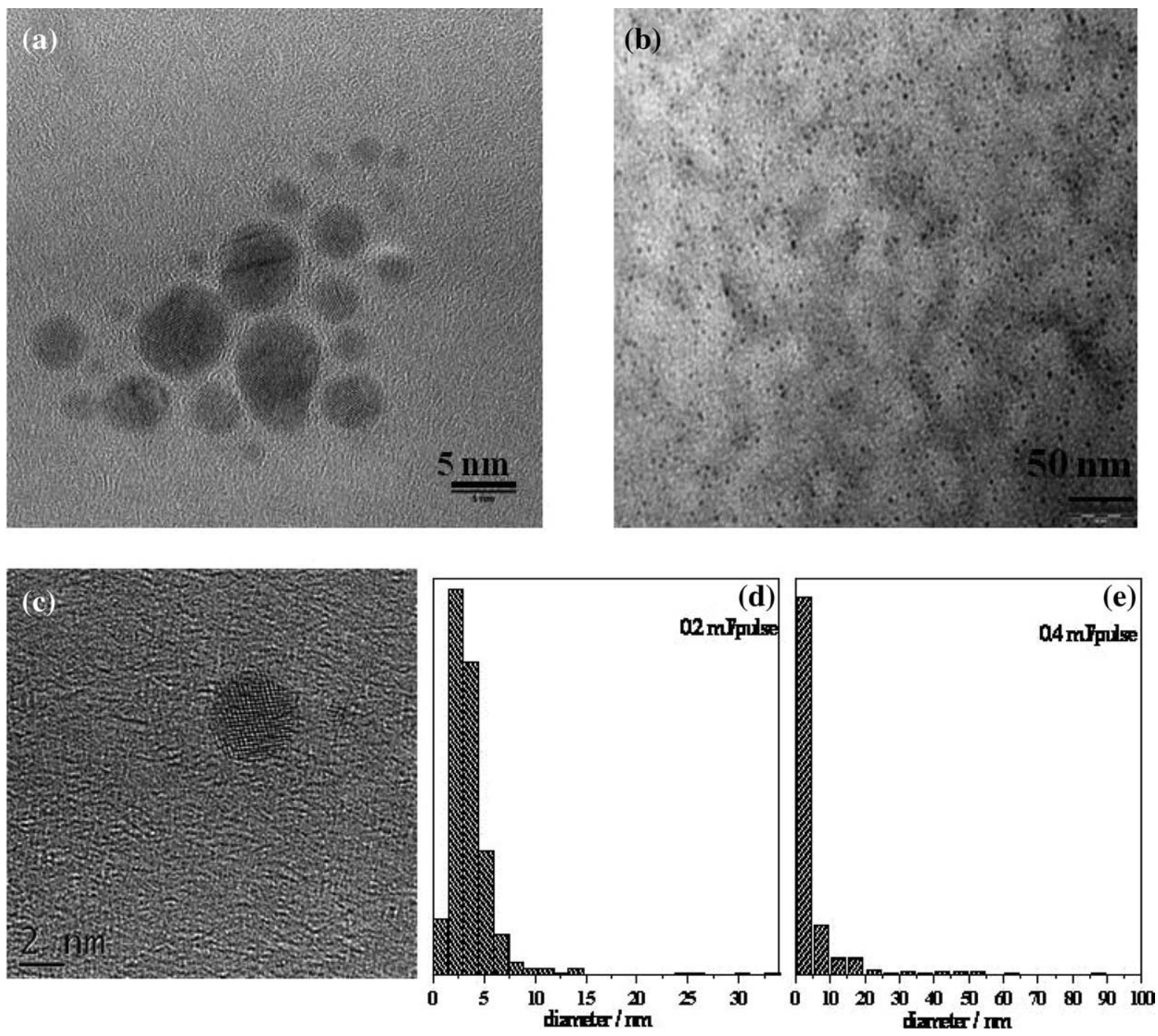

Fig. 2. HR-TEM (a), low-resolution-TEM, (b) and crystal structure (c) of the formed Au NPs by laser ablation of $\mathrm{Au}$ solid target in pure deionized water using pulses with fs duration, wavelength $800 \mathrm{~nm}$, and energy $0.2 \mathrm{~mJ} /$ pulse. Size distribution of Au NPs produced with pulses of energy $0.2 \mathrm{~mJ} / \mathrm{pulse}(\mathbf{d})$ $0.4 \mathrm{~mJ} / \mathrm{pulse}(\mathbf{e})$.

of the colloidal NPs, the maximum shifting towards longer wavelengths for bigger nanoparticles (Kreibig and Vollmer, 1995; Link and El-Sayed, 1999a). In the literature, the absorption peak of Au NPs, has been reported at $\sim 520 \mathrm{~nm}$ for NP-sizes $\sim 20 \mathrm{~nm}$ (Link and El-Sayed, 1999b), and, using the method presented herein, at $\sim 540-550 \mathrm{~nm}$ for NPs with sizes extended over the range 3-30 nm (Kabashin and Meunier, 2003).

In our case, although the absorption peaks appear at wavelengths greater than $530 \mathrm{~nm}$, characteristic for big nanoparticles $(>20 \mathrm{~nm})$, the TEM images and the histograms originating from their analysis shown in Figures 2 and 3 , demonstrate that the vast majority of the produced NPs have very small average sizes $(\sim 2.5 \mathrm{~nm})$ but a small percentage of larger NPs are always present. Increasing the laser energy from 0.2 to $0.8 \mathrm{~mJ} /$ pulse we observe that even though the majority of NPs remain small, the size of the largest NPs increases from $\sim 35 \mathrm{~nm}$ to more than $100 \mathrm{~nm}$. Indeed, the red shift of about $10 \mathrm{~nm}$ in the UV-Vis spectra, for the high laser energies is an extra evidence for the increasing size of the largest NPs. We assume that the large NPs are responsible for the red shift of the absorption spectra towards longer wavelengths, because even though their population is very small, their overall volume is considerably higher than the one of the small NPs. Indeed, an estimation of the volume ratio of the small over the large $\operatorname{NPs}\left(V_{\text {small }} / V_{\text {large }}\right)$ was done for the solutions produced at energy $0.2 \mathrm{~mJ} /$ pulse, and it was found to be $10^{-1}$. For the estimation of $V_{\text {small }}$ we added up the volumes of the NPs with diameters up to $15 \mathrm{~nm}$, calculating their percentage (99\%) in the histograms, as the one presented of Figure 2 . We consider that these NPs contribute to the UV-vis spectra at wavelengths less than $520 \mathrm{~nm}$. Similarly, for the estimation of $V_{\text {large }}$, we added up the volumes of the NPs with diameters between 15 and $35 \mathrm{~nm}$, taking under account their percentage to the statistics (1\%). 

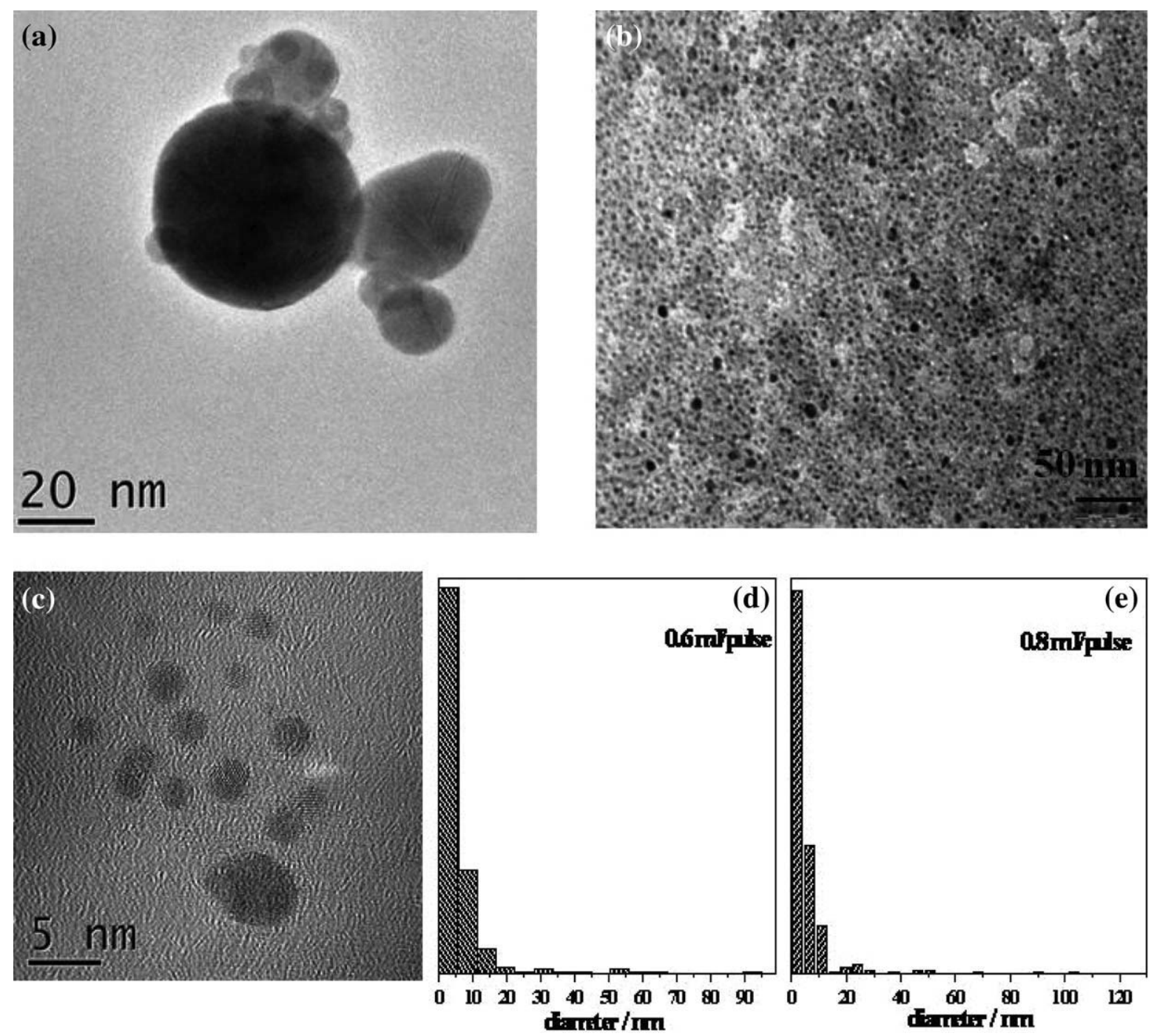

Fig. 3. HR-TEM (a), low-resolution-TEM (b), and crystal structure (c) of the formed Au NPs by laser ablation of Au solid target in pure deionized water using pulses with fs duration, wavelength $800 \mathrm{~nm}$, and energy $0.8 \mathrm{~mJ} /$ pulse. Size distribution of Au NPs produced with pulses of energy $0.6 \mathrm{~mJ} /$ pulse (d) 0.8 $\mathrm{mJ} /$ pulse $(\mathbf{e})$.

The contribution of these NPs to the UV-Vis spectra is at wavelengths higher than $520 \mathrm{~nm}$. The volume ratio of the small over the large NPs $\left(V_{\text {small }} / V_{\text {large }}\right)$ becomes even smaller for the higher laser energy regimes. The fact that NP populations with different sizes contribute to the absorption may also explain the broadening of the spectra.

The TEM results presented in Figures 2 and 3 give further details on the produced NPs. In particular, in Figures $2 \mathrm{a}, 2 \mathrm{~b}, 3 \mathrm{a}$, and $3 \mathrm{~b}$ a are shown characteristic high- and low-resolution TEM images of NPs produced by fs laser ablation using low energy pulses $(0.2 \mathrm{~mJ} /$ pulse) and high energy pulses $(0.8 \mathrm{~mJ} / \mathrm{pulse})$, respectively, at $800 \mathrm{~nm}$. The formed NPs for all energies are crystalline, exhibiting the fcc Au structure, as can be seen in Figure 2c and 3c. In Table 1 is presented the lattice constant measured from the HRTEM images, and the comparison with the value expected from fcc $\mathrm{Au}$, considering the (111), (200), and (220) reflections
TABLE 1. Lattice constants measured from the HR-TEM images of the formed Au NPs by laser ablation of solid Au target using pulses with $\mathrm{fs}$ duration, wavelength $800 \mathrm{~nm}$ and energies $0.2,0.4,0.6$, and $0.8 \mathrm{~mJ} /$ pulse in pure deionized water

\begin{tabular}{|c|c|c|c|}
\hline$A u f c c a_{0}(\AA)$ & $\begin{array}{c}\text { Energy of laser } \\
\text { irradiation for each } \\
\text { sample (mJ/pulse) }\end{array}$ & $a_{0}( \pm$ error $)(\AA)$ & Deviation (\%) \\
\hline 4.08 & $\begin{array}{l}0.2 \\
0.4 \\
0.6 \\
0.8\end{array}$ & $\begin{array}{l}3.97( \pm 0.10) \\
3.96( \pm 0.11) \\
3.99( \pm 0.10) \\
4.05( \pm 0.11)\end{array}$ & $\begin{array}{l}-2 \\
-3 \\
-2 \\
-1\end{array}$ \\
\hline
\end{tabular}

that were visible depending on the particles orientation. The differences of few percent are below the error estimated from the standard deviation on the measurement (about 3\%).

Next, the colloidal Au solutions were produced by solid $\mathrm{Au}$ target-laser ablation using ns pulses of 532 $\mathrm{nm}$ wavelength, and energies $0.4,0.9,3.0,5.2$, and 
$7.5 \mathrm{~mJ} /$ pulse, respectively. In Figure 4 are presented their UV-vis absorption spectra. We observe that for all spectra originating from the different solutions the maximum absorption peak is at $\sim 517 \mathrm{~nm}$ which is an indication for small nanoparticle sizes, around $\sim 9 \mathrm{~nm}$ (Link and El-Sayed, 1999b).

These remarks are in agreement with the conclusions obtained by the analysis of the above NP-solutions with low resolution TEM, presented in Figure 5. As can be seen by the images, and by the size distribution-histograms that derive from quantitative image analysis, as the energy of the laser pulses increases up to $5.2 \mathrm{~mJ} /$ pulse, the produced NPs have increasing av-

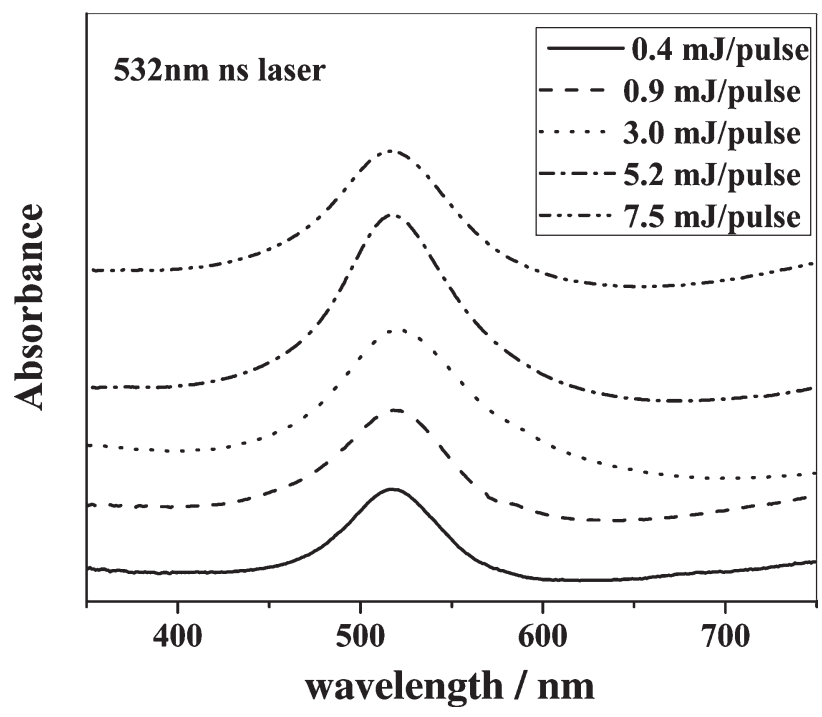

Fig. 4. UV-Vis absorption spectra of the produced Au NPs by laser ablation of solid Au target using pulses with ns duration, wavelength $532 \mathrm{~nm}$, and energies $0.4,0.9,3.0,5.2$, and $7.5 \mathrm{~mJ} / \mathrm{pulse}$. erage size (from 3.5 to $10 \mathrm{~nm}$ ) with narrow size distributions. When the energy is increased further up to $7.5 \mathrm{~mJ} /$ pulse the average size of the produced NPs decreases again down to $4.5 \mathrm{~nm}$. The latter result is in agreement with the one by Mafuné et al. (2001a) for NPs produced at high laser energies by ns laser ablation at $532 \mathrm{~nm}$.

Finally, in Figure 6 are shown four TEM images of a solution prepared using ns pulses at 1,064 nm, and laser energy $11.5 \mathrm{~mJ} /$ pulse. We observed that under these conditions are produced simultaneously NPs, fragments, and nanorods of different aspect ratios. Experiments in progress show that the percentage of these populations can be changed by varying the laser energy.

\section{DISCUSSION}

For a vast variety of applications, solutions of NPs of different sizes and narrow size distributions are prepared using diverse experimental techniques. In this work, production of $\mathrm{Au}$ NPs with different average sizes and size distributions, into ultra pure water is reported. The NPs were formed after the ablation of a solid Au target using laser pulses with tuned characteristics. In particular, were used pulses of fs duration at $800 \mathrm{~nm}$, and pulses of ns duration at 1,064 and 532 $\mathrm{nm}$. The experimental conditions were chosen carefully, in order to have comparable actual laser-Au target interaction time $\left(\sim 10^{-6} \mathrm{~s}\right)$ and energies for both pulse durations used ( $\mathrm{ns}$ and fs), so as to be possible a straightforward assessment of the effect of the pulse duration.

For the fs laser ablation experiments we optimized the conditions regarding focusing of the laser beam with respect to the surface target, and we varied the laser energy. It was shown that for laser energies from 0.2 to $0.8 \mathrm{~mJ} /$ pulse, most of the produced NPs have very small sizes $\sim 2.5 \mathrm{~nm}$, while a small percentage of dispersed larger NPs from up to $35 \mathrm{~nm}$ to more than
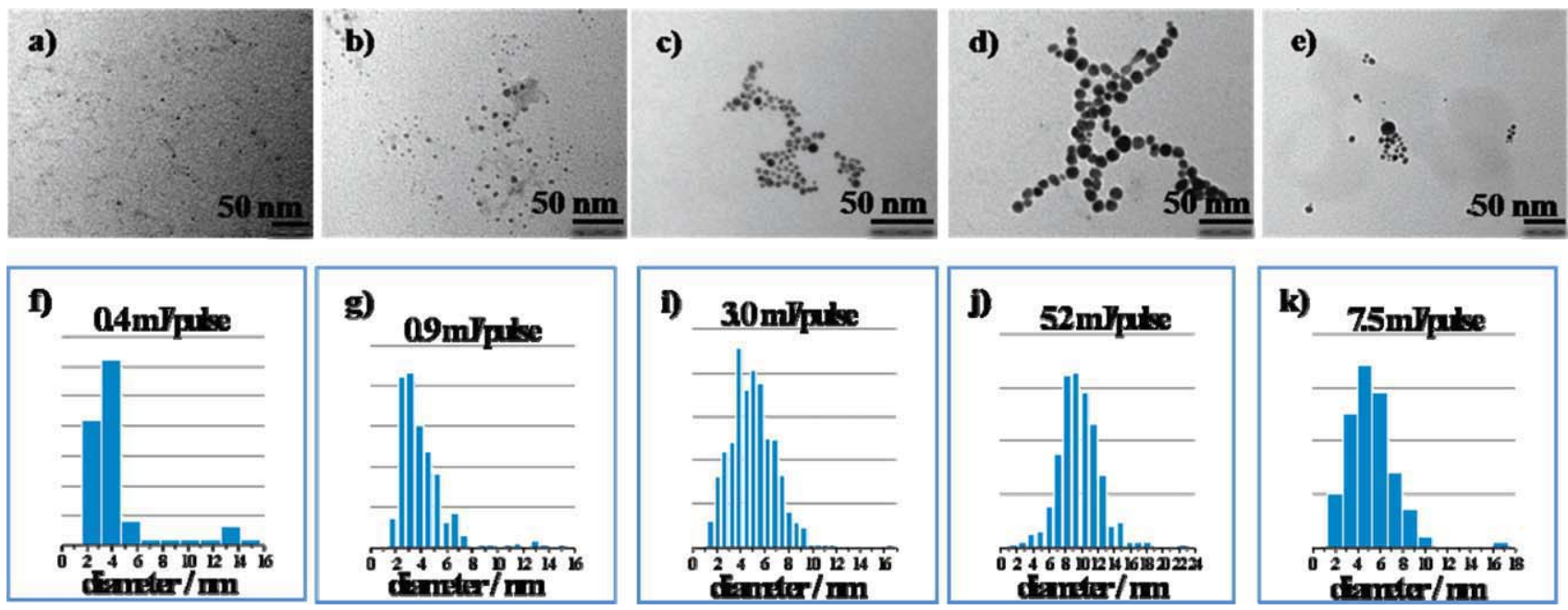

Fig. 5. Low-resolution TEM of the Au NPs formed by laser ablation of Au solid target in pure deionized water using pulses with ns duration, wavelength $532 \mathrm{~nm}$, and energy $0.4(\mathbf{a}), 0.9$ (b), 3.0 (c), 5.2 (d), and $7.5(\mathbf{e}) \mathrm{mJ} /$ pulse. Size distribution of Au NPs produced with pulses of energy $0.4(\mathbf{f}), 0.9(\mathbf{g}), 3.0(\mathbf{i})$, $5.2(\mathbf{j})$, and $7.5 \mathrm{~mJ} /$ pulse $(\mathbf{k})$. [Color figure can be viewed in the online issue, which is available at wileyonlinelibrary.com.] 

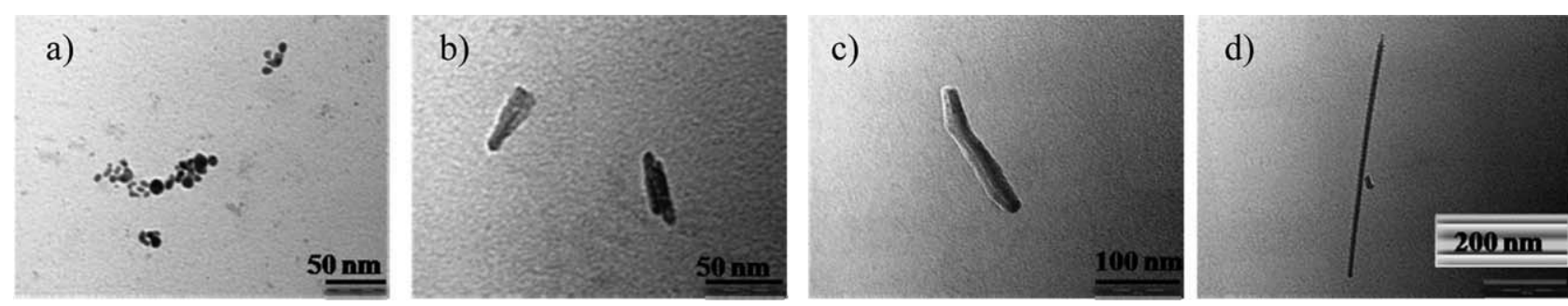

Fig. 6. Low-resolution TEM of the formed Au nanoparticles (a), fragments (b), and nanorods (c), (d) in different aspect ratios, produced by laser ablation of Au solid target in pure deionized water using pulses with ns duration, wavelength $1,064 \mathrm{~nm}$, and energy $11.5 \mathrm{~mJ} / \mathrm{pulse}$.

$100 \mathrm{~nm}$, is always present. These findings are in agreement with the two basic studies of $\mathrm{Au}$ fs laser ablation into pure water, reporting small average sizes for low energies (Kabashin and Meunier, 2003), and laser beam focusing below the surface target (Sylvestre et al., 2005). In these works, two NPs distributions; one high with small average sizes and one lower but prominent with larger sizes are reported. We accomplished by optimizing the above mentioned conditions in our setup, to produce NPs with extremely small average sizes $\sim 2.5 \mathrm{~nm}$ and to minimize but not eliminate the undesired larger NPs population. Experiments are in progress involving the production of Au NPs in different polymer solutions, in order to examine the possible effect of the polymer on the size of the NPs, and to prepare nanocomposite materials with enhanced properties.

So far, most of the reported works using ns pulses for laser ablation of $\mathrm{Au}$ in water, usually focus the laser beam onto the surface of the target and, they mention production of NPs in the range of $10-30 \mathrm{~nm}$, nanodiscs with diameters $\sim 20-60 \mathrm{~nm}$ or elongated NPs (Compagnini et al., 2002; Dolgaev et al., 2002; Simakin et al., 2001) for a wide range of fluences. In this work, on the other hand, we used the same focusing conditions as for the fs-pulsed laser ablation experiments (laser beam focusing below the surface target). In the case of the ns laser ablation, a greater range of laser energies was used, reaching values one order of magnitude higher than in the case of the fs laser ablation. We were able to produce small Au NPs $<10 \mathrm{~nm}$ using ns pulses into ultra pure water and, according to our knowledge this result is reported herein for the first time. Increasing the energy of the laser from 0.4 to $5.2 \mathrm{~mJ} / \mathrm{pulse}$, the average NPs size increases monotonically from $\sim 3.5$ to $10 \mathrm{~nm}$, a result that demonstrates the potentiality of the technique for tuning the NPs sizes. When the energy is increased above the value of $5.2 \mathrm{~mJ} /$ pulse, it was found that the average NPs size starts again decreasing. This result can be explained by a photofragmentation process proposed by (Mafune et al., 2001a) for high laser energies, in which the photon energy during single ns laser pulses is readily converted to heat into the internal modes of the NPs inducing their fragmentation. Similarly, (Takami et al., 1999) reported that irradiation of gold NPs with ns laser pulses of high energy at $532 \mathrm{~nm}$ leads to further NPfragmentation, mentioning slow heat transfer of the deposited laser energy into the surrounding solvent.

Summarizing, using fs laser ablation we observed simultaneous production of very small NPs and of a small percentage of bigger particles. The latter increase their sizes by increasing the laser energy. On the other hand, using ns laser ablation we managed to produce small NPs with monodispersed distributions that increase their average size monotonically from $\sim 3.5$ to $10 \mathrm{~nm}$, by increasing the incident energy. This difference is possibly attributed to the different ablation mechanisms occurring using fs and ns lasers pulses, due to the higher-peak powers of the first. Indeed, Henley et al. (2005) studying the ejected plume upon ablation of graphite by fs pulses, observed two shock waves propagating from the target. The first one is associated with an initial burst of highly energetic/ electronically excited ablated components, indicative of an enhanced fraction of nonthermal ejection, and the second one is associated with subsequently ejected slower moving atoms and clusters. In the same study, graphite ablation by ns pulses generated a plume that was continuously spread due to thermal expansion. The ablation of fast monomers followed by the production of clusters and chains is also reported in a theoretical work on fs ablation on graphite (Jeschke et al., 2001). This mechanism can explain the appearance of very big $\mathrm{Au} \mathrm{NPs}$ together with the small ones, in our experiments of laser ablation of an Au target with fs pulses.

Finally, in the case of the ablation of the Au solid target with ns laser pulses at $1,064 \mathrm{~nm}$, the whole procedure appears to be more complicated since at the same time are produced NPs, fragments, and nanorods with various aspect ratios. At the moment experiments are in progress trying to optimize the conditions that favor the anisotropic growth in order to produce exclusively nanorods in pure water.

In conclusion, in this work is reported the production of extremely small Au NPs in water, minimizing but not eliminating the existence of larger NPs, using the ablation technique of a solid $\mathrm{Au}$ target with laser pulses of fs duration. For this pulse duration, the best results were achieved for the smallest energy used $(0.2$ $\mathrm{mJ} /$ pulse). On the top, is demonstrated the potentiality of the technique to fine tune the size of small produced NPs from 3 to $10 \mathrm{~nm}$ in water, always having monodispersed distributions, using ns pulses with increasing energy from 0.4 to $5.2 \mathrm{~mJ} /$ pulse.

\section{ACKNOWLEDGMENT}

The authors thank Benedetta Antonazzo, technician of CNR-NNL. 


\section{REFERENCES}

Alivisatos AP. 1996. Perspectives on the physical chemistry of semiconductor nanocrystals. J Phys Chem 100:13226-13239.

Brus LE. 1991. Quantum crystallites and nonlinear optics. Appl Phys A 53:465-474.

Besner S, Kabashin AV, Winnik F M and Meunier M. 2008. Ultrafast laser based "green" synthesis of non-toxic nanoparticles in aqueous solutions. Appl Phys A 93:955-959.

Besner S, Kabashin AV, and Meunier M. 2006. Fragmentation of colloidal nanoparticles by femtosecond laser-induced supercontinuum generation. Appl Phys Lett 89:233122-1-233122-3.

Bozon-Verduraz F, Brayner R, Voronov VV, Kirichenko NA, Simakin AV, Shafeev GA. 2003. Production of nanoparticles by laser-induced ablation of metals in liquids. Quantum Electron 33:714-720.

Compagnini G, Scalisi AA, Puglisi O. 2002. Ablation of noble metals in liquids: A method to obtain nanoparticles in a thin polymeric filmy. Phys Chem Chem Phys 4:2787-2791.

Compagnini G, Scalisi AA, Puglisi O. 2006. Spectroscopic characterization of core and shell regions in monolayer protected gold nanoparticles. Surf Sci 600:L1-L5.

Dolgaev SI, Simakin AV, Voronov VV, Shafeev GA, Bozon-Verduraz F. 2002. Nanoparticles are produced by laser ablation of solids in liquid environment. Appl Surf Sci 186:546-551.

Henglein A. 1989. Small-particle research: Physicochemical properties of extremely small colloidal metal and semiconductor particles. Chem Rev 89:1861-1873.

Henglein A. 1993. Physicochemical properties of small metal particles in solution: "Microelectrode" reactions, chemisorption, composite metal particles, and the atom-to-metal transition. J Phys Chem 97:5457-5471.

Henley SJ, Carey JD, Silva SRP, Fuge GM, Ashfold MNR, Anglos D. 2005. Dynamics of confined plumes during short and ultrashort pulsed laser ablation of graphite. Phys Rev B 72:205413-1-205413-13.

Jeschke HO, Garcia ME, Bennemann KH. 2001. Theory for the ultrafast ablation of graphite films. Phys Rev Lett 87:015003-1-015003-4.
Kabashin AV, Meunier M. 2003. Synthesis of colloidal nanoparticles during femtosecond laser ablation of gold in water Synthesis of colloidal nanoparticles during femtosecond laser ablation of gold in water. J Appl Phys 94:7941-7943.

Kreibig U, Vollmer M. 1995. Optical properties of metal cluster. Berlin: Springer.

Link S, El-Sayed MA. 1999a. Spectral properties and relaxation dynamics of surface plasmon electronic oscillations in gold and silver nanodots and nanorods. J Phys Chem B 103:8410-8426.

Link S, El-Sayed MA. 1999b. Size and temperature dependence of the plasmon absorption of colloidal gold nanoparticles. J Phys Chem B 103:4212-4217.

Mafune' F, Kohno J, Takeda Y, Kondow T. 2001a. Dissociation and aggregation of gold nanoparticles under laser irradiation. J Phys Chem B 105:9050-9056.

Mafune' F, Kohno J, Takeda Y, Kondow T. 2001b. Formation of gold nanoparticles by laser ablation in aqueous solution of surfactant. J Phys Chem B 105:5114-5120.

Petersen S, Jakobi J, Hörtinger A, Barcikowski S. 2009. In-situ conjugation-Tailored nanoparticle-conjugates by laser ablation in liquids. J Laser Micro/Nanoeng 4:71-74.

Ready JF, Farson DF, editors. 2001. LIA Handbook of laser materials processing. Heidelberg: Springer.

Sibbald MS, Chumanov G, Cotton TM. 1996. Reduction of cytochrome $c$ by halide-modified, laser-ablated silver colloids. J Phys Chem 100:4672-4678.

Simakin AV, Voronov VV, Shafeev GA, Brayner R, Bozon-Verduraz F 2001. Nanodisks of Ag and Au produced by laser ablation into liquid environment. Chem Phys Lett 348:182-186.

Sylvestre JP, Kabashin AV, Sacher E, Meunier M. 2005. Femtosecond laser ablation of gold in water: Influence of the laser-produced plasma on the nanoparticle size distribution. Appl Phys A 80:753758 .

Takami A, Kurita H, Koda S. 1999. Laser-induced size reduction of noble metal particles. J Phys Chem B 103:1226-1232. 